\title{
A!
}

This is an electronic reprint of the original article.

This reprint may differ from the original in pagination and typographic detail.

Ruan, Zhongyuan; Iniguez, Gerardo; Karsai, Márton; Kertész, János

\section{Kinetics of Social Contagion}

Published in:

Physical Review Letters

DOI:

10.1103/PhysRevLett.115.218702

Published: 01/01/2015

Document Version

Publisher's PDF, also known as Version of record

Please cite the original version:

Ruan, Z., Iniguez, G., Karsai, M., \& Kertész, J. (2015). Kinetics of Social Contagion. Physical Review Letters, 115(21), 1-5. [218702]. https://doi.org/10.1103/PhysRevLett.115.218702

This material is protected by copyright and other intellectual property rights, and duplication or sale of all or part of any of the repository collections is not permitted, except that material may be duplicated by you for your research use or educational purposes in electronic or print form. You must obtain permission for any other use. Electronic or print copies may not be offered, whether for sale or otherwise to anyone who is not an authorised user. 


\title{
Kinetics of Social Contagion
}

\author{
Zhongyuan Ruan, ${ }^{1,2}$ Gerardo Iñiguez, ${ }^{3,4}$ Márton Karsai, ${ }^{5}$ and János Kertész ${ }^{1,2,4, *}$ \\ ${ }^{1}$ Center for Network Science, Central European University, H-1051 Budapest, Hungary \\ ${ }^{2}$ Institute of Physics, Budapest University of Technology and Economics, H-1111 Budapest, Hungary \\ ${ }^{3}$ Centro de Investigación y Docencia Económicas, Consejo Nacional de Ciencia y Tecnología, O1210 México D.F., Mexico \\ ${ }^{4}$ Department of Computer Science, Aalto University School of Science, FI-00076 AALTO, Finland \\ ${ }^{5}$ Laboratoire de l'Informatique du Parallélisme, INRIA-UMR 5668, IXXI, ENS de Lyon, 69364 Lyon, France \\ (Received 30 May 2015; revised manuscript received 17 October 2015; published 20 November 2015)
}

Diffusion of information, behavioral patterns or innovations follows diverse pathways depending on a number of conditions, including the structure of the underlying social network, the sensitivity to peer pressure and the influence of media. Here we study analytically and by simulations a general model that incorporates threshold mechanism capturing sensitivity to peer pressure, the effect of "immune" nodes who never adopt, and a perpetual flow of external information. While any constant, nonzero rate of dynamically introduced spontaneous adopters leads to global spreading, the kinetics by which the asymptotic state is approached shows rich behavior. In particular, we find that, as a function of the immune node density, there is a transition from fast to slow spreading governed by entirely different mechanisms. This transition happens below the percolation threshold of network fragmentation, and has its origin in the competition between cascading behavior induced by adopters and blocking due to immune nodes. This change is accompanied by a percolation transition of the induced clusters.

DOI: 10.1103/PhysRevLett.115.218702

PACS numbers: 89.65.Ef, 64.60.ah, 89.75.Hc

There are remarkable analogies between the social contagion of information, behavioral patterns or innovation, and some physical or epidemic spreading processes, where global phenomena emerge through the diffusion of microscopic states [1-4]. All evolve in networks with nodes characterized by relevant state variables, and links that represent direct interactions between nodes. In biological systems epidemics are driven by binary interactions that lead to the emergence of simple contagion phenomena [1]. Social diffusion processes are usually characterized by complex contagion mechanisms, where node states are determined by comparing individual thresholds with all neighbor states [2,5-8]. This property, capturing the effect of peer pressure and commonly assumed in social spreading phenomena $[9,10]$, has consequences on the dynamics and the final outcome of the social contagion process. Moreover, the theoretical approach to these systems has much in common [1,6,11], which greatly helps us to understand their behavior.

Models employing threshold mechanisms mostly focus on cascading phenomena where, under some circumstances, a macroscopic fraction of nodes in the network is converted rapidly due to microscopic perturbations. This approach is motivated by earlier social theories $[9,12]$ and has been implemented by Watts in an elegant model of cascading behavior [6]. Watts showed that a global cascade (occupying a macroscopic fraction of the network and induced by local perturbations) can occur due to the interplay between network structure and individual thresholds. He further identified the phase with a nonzero probability of global cascades in the space $(\phi, z)$ of the average threshold $\phi$ of nodes and the average degree $z$ of the network.
While the relevance of this model is indisputable $[6,11$, 13-22], its limitations become clear from real social spreading data. The Watts model focuses on the (instantaneous) emergence of global cascades triggered by single local perturbations, while there are empirical examples where threshold mechanisms do play a role yet global adoption phenomena emerge through other scenarios. In reality, global adoption is often not induced by microscopic perturbations but by a larger fraction of people [21]. Moreover, decisions of individuals depend on external impulses arriving from mass media or advertising [23], resulting in a perpetual stochastic perturbation. In addition, there are individuals entirely reluctant to adopt. Furthermore, the Watts criterion for macroscopic adoption is purely deterministic, coded in the network structure, threshold distribution, and perturbation site-it does not concern time, which is clearly a feature of empirical stochastic processes of adoption spreading.

Here we present a general threshold-driven model of social contagion phenomena that captures various spreading scenarios, ranging from cascading behavior to dynamically evolving nonexplosive patterns, and sheds light to the different kinetics behind them (Fig. 1). Motivated by empirical observations [24], we extend Watts' threshold model by considering blocked nodes immune to social influence and discuss their effect on cascade formation. In addition, we introduce spontaneous adopters with a constant rate, and present approximate analytical and numerical results regarding our model. In particular, we study how the kinetics of spreading changes for an increasing density of blocked nodes. We aim at the simplest possible but 
(a)
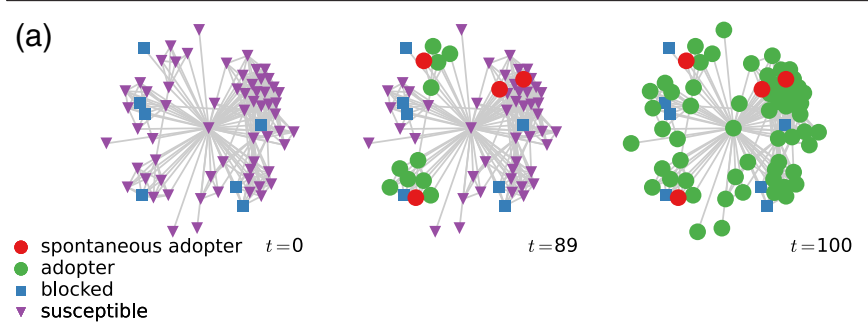

(b)
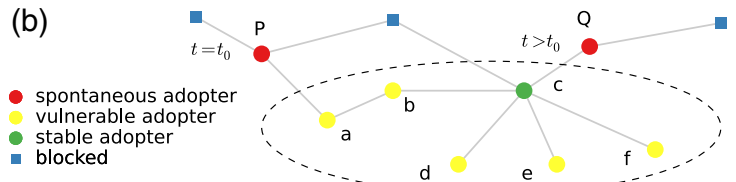

vulnerable adopter

- stable adopter

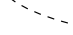

-

FIG. 1 (color online). (a) Numerical simulation of a general threshold model over an empirical network, with adoption threshold $\phi=0.2$, rate of spontaneous adopters $p=0.0005$, and fraction of blocked nodes $r=0.1$. The network is an ego sample of Facebook friendships with size $N=96$ and average degree $z=10.63$ [25]. Susceptible nodes adopt spontaneously with rate $p$ or after a fraction $\phi$ of their neighbors has adopted, while blocked nodes never adopt. (b) Schematic illustration of the spreading process. At $t=t_{0}$ node $P$ spontaneously becomes an adopter, "infecting" nodes a and $\mathrm{b}$. When $Q$ adopts, it induces the adoption of nodes $\mathrm{c}-\mathrm{f}$. Nodes inside the ellipse constitute an induced cluster of adoption.

sufficiently general extension of earlier threshold models [6,17-22] with a minimal set of states and transitions necessary to describe various real scenarios of social spreading phenomena. The introduction of further states, secondary adoption, or other decision-making mechanisms is left as a further challenge, since our aim here is to model generic cascades of primary adoption.

In Watts' threshold model [6], all nodes are initially in a susceptible state 0 , except for a single adopter seed in state 1 . The process evolves as each node with degree $k$ changes its state from 0 to 1 if a fraction $\phi$ of its neighbors have adopted before. Since nodes cannot change their state after exposure, the system evolves towards a state where no further adoptions are possible. The emergence of a global cascade depends on the degree distribution $p_{k}$ of the network, the distribution $p_{\phi}$ of individual thresholds, and the initial seed. The condition for a global cascade is the existence of a percolating component of vulnerable nodes with thresholds $0<\phi \leq 1 / k$ (who need one adopting neighbor before exposure) connected to the seed. This percolating vulnerable tree is quickly converted after adoption of the seed and may trigger further adoption of stable nodes with thresholds $\phi>1 / k$ (who need more than one adopting neighbor to adopt). Assuming an Erdős-Rényi (ER) random network [26] and a single adopter seed, there is a phase boundary in $(\phi, z)$ space encompassing a regime where global cascades occur [Fig. 2(a)]. The properties of this cascading regime have been investigated for the case of heterogeneous thresholds, different network topologies $[6,11]$, and variable seed size $[16,21]$.
Empirical studies, however, support the intuition that some individuals in society may refuse to adopt technological innovations for various reasons-due to another favorite product, aversion towards a firm, or some criticism on principle [24]. Such individuals will never be exposed, irrespective of the state of their neighbors [27]. To consider this behavioral pattern in our model, we block the adoption of a fraction $r$ of randomly selected nodes in the network. These nodes do count when their neighbors consider the decision to adopt, and thus will make it harder for neighbors to fulfil the threshold criterion.

Accordingly, the original Watts model corresponds to $r=0$, while for $r>0$ the phase diagram changes. Even in the presence of blocked nodes, macroscopic spreading is still determined by the static criterion of the existence of a global vulnerable cluster, and thus a generating function technique [6] can be applied [28] [29]. Assuming a single threshold $\phi$ and an ER network with average degree $z$, the condition for the emergence of a macroscopic cascade is

$$
(1-r) e^{-z} \sum_{k=2}^{k_{c}} \frac{z^{k}}{(k-2) !}-z=0,
$$

with $k_{c}=\lfloor 1 / \phi\rfloor$. Because of the factor $1-r$, the introduction of blocked nodes shrinks the region in $(\phi, z)$ space where global cascades develop, in good agreement with numerical simulations [Figs. 2(c) and 2(d)].

While blocked nodes hinder the spreading process, there are reasons other than social influence that could motivate
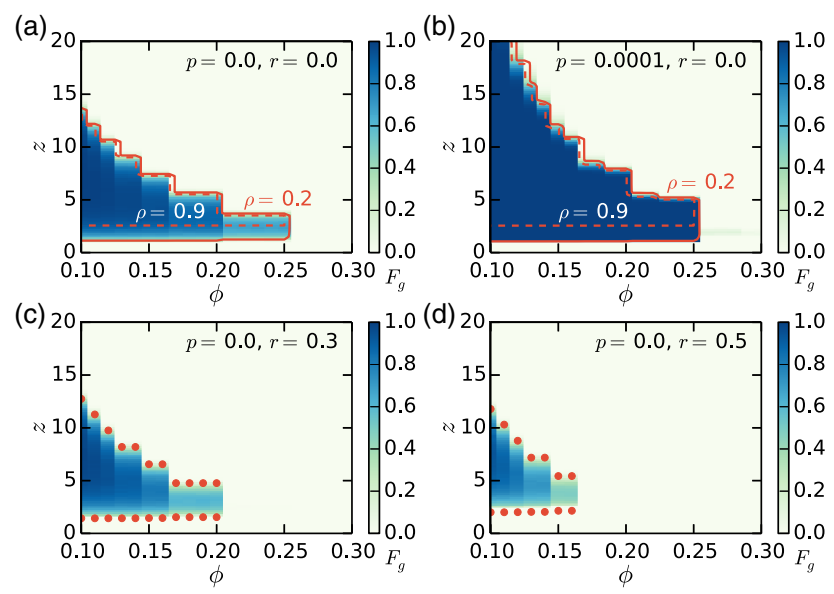

FIG. 2 (color online). Frequency $F_{g}$ of global cascades as a function of node threshold $\phi$ and network average degree $z$ at an intermediate time $t=100$, for varying $p$ and $r$. (a)-(b) As $p$ increases, the region that allows global cascades of adoption grows in size. Its boundary is well approximated by a cutoff in the fraction of adopters $\rho$ as calculated by Eq. (5). (c)-(d) Conversely, an increasing fraction of blocked nodes shrinks the global cascade regime. Dots show the boundary of this regime according to Eq. (1). Simulations correspond to an ER network with $N=10^{4}$ and are averaged over $10^{4}$ realizations. 
individuals to adopt a social pattern, like external influence from mass media. This spontaneous adoption has been studied theoretically by introducing a given density of adopters at the outset of the Watts model [21]. However, spontaneous adopters may get active at any time during a real social contagion. Thus, we include a stochastic dynamics where a susceptible node may become adopter with rate $p$ at any time, irrespective of the status of its neighbors.

Considering both extensions, we have a threshold-driven dynamics with three node states: blocked, susceptible, and adopter (Fig. 1). At the outset, all nodes are susceptible except for a fraction $r$ that remains blocked. At each time step of the simulation, a randomly selected, susceptible node $i$ adopts spontaneously with probability $p$; otherwise, it adopts if at least a fraction $\phi$ of its neighbors has already adopted. If $r=0$ and $p>0$ all nodes will eventually adopt [Fig. 3(a)], following a kinetics reminiscent of the approach to a unique ground state in a physics system. On the other hand, if we introduce quenched randomness and stochastic perturbations $(r, p>0)$, our model allows various temporal regimes and a transition from rapid to slow spreading dynamics.

Our threshold model can be studied analytically by extending the framework of approximate master equations (AMEs) for monotone binary-state dynamics developed by Gleeson [14-16,30], where the transition rate between susceptible and adoption states only depends on the number $m$ of neighbors that have already adopted. We ignore

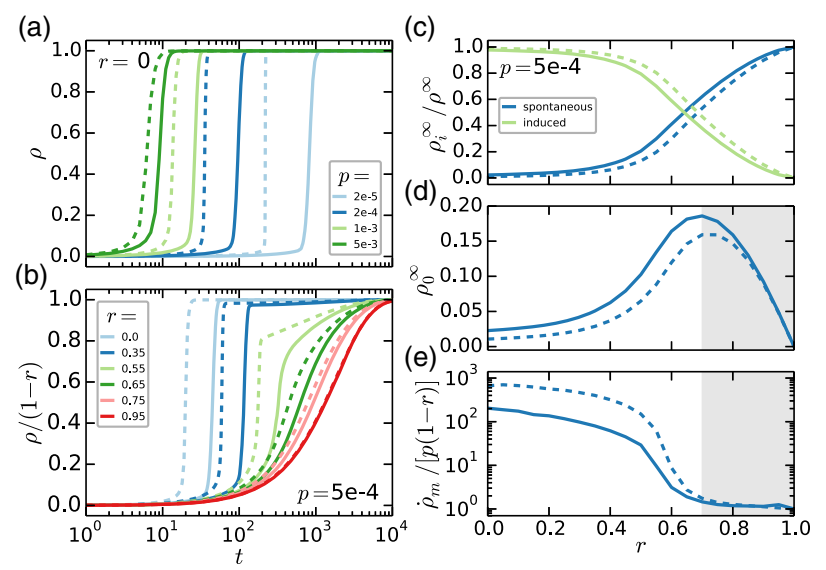

FIG. 3 (color online). Numerical simulations and analytical approximation of the threshold model for $z=7$ and $\phi=0.2$ (continuous and dotted lines, respectively). (a) Fraction of adopters $\rho$ as a function of time for varying $p$ and fixed $r$. (b) Time evolution of the normalized adoption density $\rho /(1-r)$ for different values of $r$ and fixed $p$. (c) Final relative density $\rho_{i}^{\infty} / \rho^{\infty}$ as a function of $r$, for both spontaneous and induced adopters ( $i=0,1$, respectively). (d) Final fraction of spontaneous adopters $\rho_{0}^{\infty}$ as a function of $r$. (e) Normalized maximum speed of spreading $\dot{\rho}_{m} /[p(1-r)]$, calculated from the derivative of $\rho(t)$. Shaded areas signal the regime of slow contagion $r>r_{\times}$. Curves correspond to $N=10^{4}$ and are averaged over $10^{3}$ realizations. topological correlations by considering a configurationmodel network with degree distribution $p_{k}$ and average degree $z$. We describe a node by the property vector $\mathbf{k}=(k, c)$, where $k=0,1, \ldots$ is its degree and $c=0,1$ its type; i.e., $c=0$ is the type of the fraction $r$ of blocked nodes, while $c=1$ is the type of all nodes that may adopt with threshold $\phi$. Moreover, $p_{\mathbf{k}}$ is a joint distribution giving the probability that a randomly selected node has property vector $\mathbf{k}$. Assuming independence between degrees and types, $p_{\mathbf{k}}=r p_{k}$ for $c=0$ and $p_{\mathbf{k}}=(1-r) p_{k}$ for $c=1$.

The rules of our model are condensed in the probability $F_{\mathbf{k}, m} d t$ that a $\mathbf{k}$-node will adopt in a small time interval $d t$, given that $m$ of its neighbors are already adopters, where,

$$
F_{\mathbf{k}, m}=\left\{\begin{array}{ll}
p & \text { if } m<k \phi \\
1 & \text { if } m \geq k \phi
\end{array}, \quad \forall m \quad \text { and } \quad k>0\right.
$$

with $F_{(k, 0), m}=0 \forall k, m$ and $F_{(0,1), 0}=p$ (for blocked and isolated nodes, respectively). The dynamics of adoption is well described by an AME for the fraction $s_{\mathbf{k}, m}(t)$ of $\mathbf{k}$-nodes that are susceptible at time $t$ and have $m=0, \ldots, k$ adopting neighbors $[14,15,30]$,

$\dot{s}_{\mathbf{k}, m}=-F_{\mathbf{k}, m} s_{\mathbf{k}, m}-\beta_{s}(k-m) s_{\mathbf{k}, m}+\beta_{s}(k-m+1) s_{\mathbf{k}, m-1}$,

where,

$$
\beta_{s}=\frac{\sum_{\mathbf{k}} p_{\mathbf{k}} \sum_{m}(k-m) F_{\mathbf{k}, m} s_{\mathbf{k}, m}}{\sum_{\mathbf{k}} p_{\mathbf{k}} \sum_{m}(k-m) s_{\mathbf{k}, m}}
$$

To reduce the dimensionality of Eq. (3) we focus on $\rho(t)$, the fraction of adopters in the network, and $\nu(t)$, the probability that a randomly chosen neighbor of a susceptible node is an adopter. We consider the ansatz $s_{\mathbf{k}, m}=$ $B_{k, m}(\nu) e^{-p t}$ for $m<k \phi$ with the binomial distribution $B_{k, m}(\nu)=\left(\begin{array}{l}k \\ m\end{array}\right) \nu^{m}(1-\nu)^{k-m}$, leading to the condition $\dot{\nu}=\beta_{s}(1-\nu)$. Then, the AME system is reduced to the pair of ordinary differential equations (see the Supplemental Material [28]),

$$
\begin{aligned}
& \dot{\rho}=h(\nu, t)-\rho, \\
& \dot{\nu}=g(\nu, t)-\nu,
\end{aligned}
$$

with initial conditions $\rho(0)=\nu(0)=0$. Here,

$$
h(\nu, t)=(1-r)\left[f_{t}+\left(1-f_{t}\right) \sum_{k} p_{k} \sum_{m \geq k \phi} B_{k, m}(\nu)\right],
$$




$$
g(\nu, t)=(1-r)\left[f_{t}+\left(1-f_{t}\right) \sum_{k} \frac{k}{z} p_{k} \sum_{m \geq k \phi} B_{k-1, m}(\nu)\right],
$$

with $f_{t}=1-(1-p) e^{-p t}$. A linear stability analysis of the reduced AME system recovers the cascade condition for $p=0$ (1) (see the Supplemental Material [28]). Moreover, the fraction of adopters $\rho(t)$ obtained by solving Eq. (5) is in considerable agreement with numerical simulations [Figs. 3(a) and 3(b)]. Since susceptible nodes adopt spontaneously with rate $p$, their fraction $\rho_{0}(t)$ in the network is approximated by

$$
\rho_{0}=p \int_{0}^{t}(1-r-\rho) d t
$$

where $\rho(t)$ follows Eq. (5) [Figs. 3(c) and 3(d)]. We denote its counterpart $\rho_{1}=\rho-\rho_{0}$ as the fraction of induced adoptions, i.e., vulnerable and stable adopters.

For $p>0$ the dynamics has a trivial asymptotic state with a final fraction of adopters $\rho^{\infty}=1-r$, however, the kinetics of the model depends on the parameters. We first focus on the frequency $F_{g}$ of global cascades (i.e., adoption reaching at least $20 \%$ of susceptible nodes [6]) and its behavior in $(\phi, z)$ space for varying $p$ and $r$. For fixed $t$ and $p>0$, there is a region where global cascades occur [Fig. 2(b)] that can be compared with the asymptotic cascade regime found for $p=0$. The boundary of this regime is well approximated by Eq. (1) for $p=0$ and by Eq. (5) for $p \geq 0$. By continuously introducing spontaneous adopters the global cascade regime expands, meaning that macroscopic adoption is eventually possible for systems with any degree and threshold. Even in the absence of a percolating vulnerable component in the network, a growing number of spontaneous adopters induces local cascades that merge due to triggered stable adoptions and finally form a giant component. This behavior is consistent with empirical observations in the online spreading of communication technologies [24].

The kinetics of spreading may change by introducing many blocked nodes. As $r$ (and thus random quenching) increases the adoption process slows down [Fig. 3(b)]. In this dynamics nodes change state in two ways: (i) via spontaneous adoption (a slow process for small $p$ ), or (ii) via induced adoption by fulfilling the threshold condition, which may lead to fast cascading behavior. For small $r$, induced adoptions dominate spreading [Fig. 3(c)] and $\rho$ grows rapidly towards $\rho^{\infty}$. On the other hand, for large $r$ adoption slows down since stable nodes have more blocked neighbors and it is difficult to fulfil their threshold condition. This slow regime is mostly driven by spontaneous adoption, as evidenced by the relatively large asymptotic fraction of spontaneous adopters $\rho_{0}^{\infty}$ [Fig. 3(d)].

Taking the ER network as an example, a giant component of susceptible nodes can only exist for $r<r *=1-1 / z[31,32]$. Then, a relevant question is whether regimes of fast and slow spreading are separated by a characteristic value $r_{\times}<r *$. One possibility is to define $r_{\times}$as the value that maximizes $\rho_{0}^{\infty}$ and for which $\rho_{0}^{\infty} \sim \rho_{1}^{\infty}$, with $\rho_{1}^{\infty}$ the final fraction of induced adopters. For $z=7$, $\phi=0.2$, and $p=0.0005$ we have $r_{\times} \approx 0.7$ and $r *=0.857$, meaning that slow spreading occurs even in susceptible networks that are not fragmented. The slow regime is further characterized by the lowest possible value in the maximum spreading speed, $\dot{\rho}_{m} \sim p(1-r)$, corresponding to the rate of spontaneous adoption at the beginning of the dynamics [Fig. 3(e)]. In other words, the time series $\rho(t)$ has an inflection point for $r<r_{\times}$and is concave for $r>r_{\times}$.

To better understand the kinetics of the crossover between spreading regimes around $r_{\times}$, we finally focus on the size distribution $P(s)$ of induced clusters, i.e., connected components of adopters disregarding spontaneous adopters [Fig. 1(b)]. For early times $P(s)$ includes small induced clusters only, indicating that a larger fraction of spontaneous adopters is crucial for global spreading in the absence of a percolating vulnerable component [Fig. 4(a)]. However, for late times the behavior of $P(s)$ differs between regimes: in the regime of rapid spreading the distribution becomes bimodal due to the appearance of a global cluster of induced adopters, while in the slow regime $\left(r>r_{\times}\right)$it remains unimodal until the end of the dynamics. Overall, the crossover between regimes seen globally in the speed of spreading (Fig. 3) is accompanied
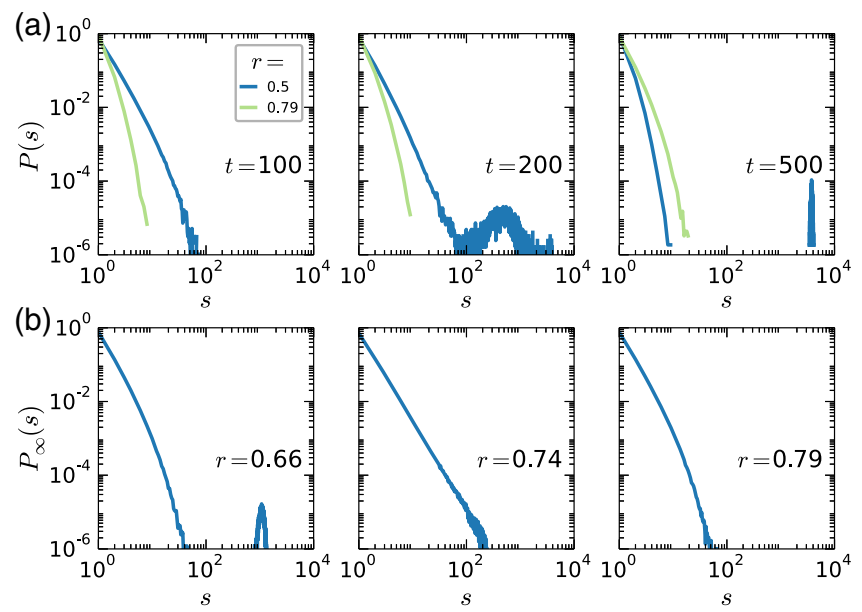

FIG. 4 (color online). (a) Size distribution $P(s)$ of induced clusters in the regimes of fast $\left(r<r_{\times}\right)$and slow $\left(r>r_{\times}\right)$ spreading, at several stages in the adoption process. In early times, $P(s)$ is unimodal and qualitatively similar in both regimes. As $t$ increases, the distribution becomes bimodal only for $r<r_{\times}$, indicating the presence of global cascades. (b) Asymptotic size distribution $P_{\infty}(s)$ of induced clusters, after $t=5000$ and for varying $r$. For $r<r_{\times}$global cascades may still develop and make $P_{\infty}(s)$ bimodal. As $r$ increases, the distribution becomes unimodal and global cascades disappear. Simulations correspond to $z=7, \phi=0.2, p=0.0005, N=10^{4}$, and are averaged over $10^{4}$ realizations. 
by an underlying, percolation-type transition revealed by the asymptotic size distribution $P_{\infty}(s)$ [Fig. 4(b)]. Indeed, in the asymptotic limit $t \rightarrow \infty$ and as $r$ increases, this distribution stops being bimodal at $r \approx 0.74 \sim r_{\times}$for the studied ER case.

The peculiarity of this dynamic percolation transition of the induced clusters is that, in contrast to the static percolation problems, it is not known a priori which node will participate in the process, as any unblocked node may become a spontaneous innovator. By analyzing the properties of this transition, we find a critical percolation point at $r_{c} \sim 0.738$ with the set of exponents $\beta=1.1, \gamma=1.0$, $\tau=2.5$, and $\nu=3.1$, which are rather close to the mean field values [33].

Our aim in this Letter has been to provide a general dynamic model of social spreading phenomena that accounts for various kinetics. Our model is designed such that it (a) is driven by threshold mechanisms capturing the role of social pressure, and (b) concerns temporal aspects of the emergence of global cascades. We generalized Watts' threshold model [6] with mechanisms of spontaneous adoption and complete reluctance to adoption, in order to further understand the temporal behavior of spreading phenomena. We have shown that, outside of the cascading regime of the Watts model, there is the possibility of global contagion mediated by spontaneous adopters. However, the speed of spreading depends strongly on the density of blocked or immune nodes. For a small fraction $r$ of blocked nodes, few spontaneous adopters enable the formation of large clusters by initiating cascades. For large $r$, spreading slows down as it is dominated by spontaneous adopters and only small cascades are generated. Our intrinsically dynamic model is able to describe various scenarios of real social contagion as well as the crossover between them, and shows a novel percolation transition of induced clusters. This model has not only the potential to explain observational data [24] but, with appropriate fitting, may help identify the character of spreading processes at an early stage, hinting in this way at possible measures to improve adoption performance. Moreover, it is possible that the consideration of blocked nodes will help understand a diversity of spreading phenomena, including related seismic or neural processes.

R. Z. acknowledges support from EU FP7 FET OPEN Grant No. 317532 (MULTIPLEX), G. I. from the Academy of Finland, and J. K. from H2020 FETPROACT-GSS CIMPLEX Grant No. 641191.

*janos.kertesz@gmail.com

[1] A. Barrat, M. Barthélemy, and A. Vespignani, Dynamical Processes on Complex Networks (Cambridge University Press, Cambridge, England, 2008).
[2] D. Easley and J. Kleinberg, Networks, Crowds, and Markets: Reasoning about a Highly Connected World (Cambridge University Press, Cambridge, England, 2010).

[3] W. Goffman and V. Newill, Nature (London) 204, 225 (1964).

[4] M. O. Jackson, Social and Economic Networks (Princeton University Press, Princeton, NJ, 2008).

[5] M. Karsai, G. Iñiguez, K. Kaski, and J. Kertész, J. R. Soc. Interface 11, 20140694 (2014).

[6] D. J. Watts, Proc. Natl. Acad. Sci. U.S.A. 99, 5766 (2002).

[7] B. Wejnert, Annu. Rev. Sociol. 28, 297 (2002).

[8] D. Centola and M. Macy, Am. J. Sociology 113, 702 (2007).

[9] M. Granovetter and R. Soong, J. Math. Sociol. 9, 165 (1983).

[10] D. Centola, Science 329, 1194 (2010).

[11] J. P. Gleeson and D. J. Cahalane, Phys. Rev. E 75, 056103 (2007).

[12] T. C. Schelling, Am. Econ. Rev. 59, 488 (1969).

[13] D. J. Watts and P. S. Dodds, Journal of consumer research 34, 441 (2007).

[14] J. P. Gleeson, Phys. Rev. X 3, 021004 (2013).

[15] J. P. Gleeson, Phys. Rev. Lett. 107, 068701 (2011).

[16] J. P. Gleeson, Phys. Rev. E 77, 046117 (2008).

[17] D. Centola, V. M. Eguíluz, and M. W. Macy, Physica (Amsterdam) 374A, 449 (2007).

[18] J. L. Payne, P. S. Dodds, and M. J. Eppstein, Phys. Rev. E 80, 026125 (2009).

[19] O. Yağan and V. Gligor, Phys. Rev. E 86, 036103 (2012).

[20] A. Nematzadeh, E. Ferrara, A. Flammini, and Y.-Y. Ahn, Phys. Rev. Lett. 113, 088701 (2014).

[21] P. Singh, S. Sreenivasan, B. K. Szymanski, and G. Korniss, Sci. Rep. 3, 2330 (2013).

[22] P. Piedrahíta, J. Borge-Holthoefer, Y. Moreno, and A. Arenas, Europhys. Lett. 104, 48004 (2013).

[23] G. Kocsis and F. Kun, Phys. Rev. E 84, 026111 (2011).

[24] M. Karsai, G. Iñiguez, R. Kikas, K. Kaski, and J. Kertész, Global contagion with local cascades: The anatomy of online adoption spreading (to be published).

[25] J. Leskovec and J. J. Mcauley, in Adv. Neural Inf. Process. Syst. (NIPS), Vol. 25, edited by F. Pereira, C. Burges, L. Bottou, and K. Weinberger (Curran Associates, Red Hook, NY, 2012), p. 539.

[26] P. Erdős and A. Rényi, Publ. Math. Inst. Hungar. Acad. Sci 5, 17 (1960).

[27] E. Yildiz, A. Ozdaglar, D. Acemoglu, A. Saberi, and A. Scaglione, ACM Trans. Econ. Comput. 1, 19 (2013).

[28] See Supplemental Material at http://link.aps.org/ supplemental/10.1103/PhysRevLett.115.218702 for the generating function approach for $p=0$ and AME formalism, which includes Ref. [29].

[29] M. E. J. Newman, S. H. Strogatz, and D. J. Watts, Phys. Rev. E 64, 026118 (2001).

[30] M. A. Porter and J. P. Gleeson, arXiv:1403.7663.

[31] D. S. Callaway, M. E. J. Newman, S. H. Strogatz, and D. J. Watts, Phys. Rev. Lett. 85, 5468 (2000).

[32] R. Cohen, K. Erez, D. ben-Avraham, and S. Havlin, Phys. Rev. Lett. 85, 4626 (2000).

[33] For notation and the related theory see D. Stauffer and A. Aharony, Introduction to Percolation Theory (Taylor and Francis, London, 1994), except that the exponent $-(1 / \nu)$ is defined here not by the scaling with the linear dimension of the system but with its size. 